\title{
Whole Genome Sequencing Reveals the Effects of Recent Artificial Selection on Litter Size of Bamei Mutton Sheep
}

\author{
Yaxin Yao ${ }^{1}$, Zhangyuan Pan ${ }^{1}$, Ran $\mathrm{Di}^{1}$, Qiuyue Liu ${ }^{1}$, Wenping $\mathrm{Hu}{ }^{1}{ }^{1}$, Xiaofei Guo ${ }^{1,2}$, Xiaoyun He ${ }^{1}$, \\ Shangquan Gan ${ }^{3}$, Xiangyu Wang ${ }^{1, *}$ and Mingxing Chu ${ }^{1, *}$
}

1 Key Laboratory of Animal Genetics, Breeding and Reproduction of Ministry of Agriculture and Rural Affairs, Institute of Animal Science, Chinese Academy of Agricultural Sciences, Beijing 100193, China; yaoyaxin0602@126.com (Y.Y.); pzq170450077@163.com (Z.P.); dirangir1@163.com (R.D.); qiuyue1983921@163.com (Q.L.); pinkyhoho@163.com (W.H.); guoxfnongda@163.com (X.G.); hedayun@sina.cn (X.H.)

2 Tianjin Institute of Animal Sciences, Tianjin 300381, China

3 State Key Laboratory of Sheep Genetic Improvement and Healthy Production, Xinjiang Academy of Agricultural and Reclamation Sciences, Shihezi 832000, China; shangquangan@163.com

* Correspondence: wangxiangyu@caas.cn (X.W.); chumingxing@caas.cn (M.C.); Tel.: +86-010-62816001 (X.W.); $+86-010-62819850$ (M.C.)

check for

updates

Citation: Yao, Y.; Pan, Z.; Di, R.; Liu, Q.; Hu, W.; Guo, X.; He, X.; Gan, S.; Wang, X.; Chu, M. Whole Genome Sequencing Reveals the Effects of Recent Artificial Selection on Litter Size of Bamei Mutton Sheep. Animals 2021, 11, 157. https://doi.org/ 10.3390/ani11010157

Received: 7 December 2020 Accepted: 6 January 2021 Published: 12 January 2021

Publisher's Note: MDPI stays neutral with regard to jurisdictional clai$\mathrm{ms}$ in published maps and institutional affiliations.

Copyright: $\odot 2021$ by the authors. Licensee MDPI, Basel, Switzerland. This article is an open access article distributed under the terms and conditions of the Creative Commons Attribution (CC BY) license (https:// creativecommons.org/licenses/by/ $4.0 /)$.
Simple Summary: Bamei mutton sheep is a Chinese domestic sheep breed developed by crossing German Mutton Merino sheep and indigenous Mongolian sheep for meat production. There is large variation in the reproductive abilities of Bamei mutton sheep. After recent artificial selection, the average lambing rate of the Bamei mutton nucleus group was over 150\%. We used the FST (Fixation Index) and XP-EHH (The Cross-Population Extended Haplotype Homozygosity) statistical approach to detect the selective sweeps between high- and low-fecundity Bamei mutton sheep groups. JUN (JUN proto-oncogene, AP-1 transcription factor subunit), ITPR3 (inositol 1,4,5-trisphosphate receptor type 3, PLCB2 (phospholipase C beta 2), HERC5 (HECT and RLD domain containing E3 ubiquitin protein ligase 5), and KDM4B (lysine demethylase 4B) were detected that are potential responsible for litter size. These observations provide a new opportunity to research the genetic variation influencing fecundity traits within a population evolving under artificial selection.

Abstract: Bamei mutton sheep is a Chinese domestic sheep breed developed by crossing German Mutton Merino sheep and indigenous Mongolian sheep for meat production. Here, we focused on detecting candidate genes associated with the increasing of the litter size in this breeds under recent artificial selection to improve the efficiency of mutton production. We selected five high- and five lowfecundity Bamei mutton sheep for whole-genome resequencing to identify candidate genes for sheep prolificacy. We used the FST and XP-EHH statistical approach to detect the selective sweeps between these two groups. Combining the two selective sweep methods, the reproduction-related genes JUN, ITPR3, PLCB2, HERC5, and KDM4B were detected. JUN, ITPR3, and PLCB2 play vital roles in GnRH (gonadotropin-releasing hormone), oxytocin, and estrogen signaling pathway. Moreover, KDM4B, which had the highest FST value, exhibits demethylase activity. It can affect reproduction by binding the promoters of estrogen-regulated genes, such as FOXA1 (forkhead box A1) and ESR1 (estrogen receptor 1). Notably, one nonsynonymous mutation (p.S936A) specific to the high-prolificacy group was identified at the TUDOR domain of KDM4B. These observations provide a new opportunity to research the genetic variation influencing fecundity traits within a population evolving under artificial selection. The identified genomic regions that are responsible for litter size can in turn be used for further selection.

Keywords: Bamei mutton sheep; whole-genome sequencing; litter size; selection signal analysis; breeding 


\section{Introduction}

Sheep were the first grazing animals bred for their meat. Mutton still has major economic value for sheep production. The domestication of sheep was initiated approximately 9000 years ago in Southwest Asia, in present-day also in Iran and Turkey [1]. Sheep husbandry began 5000-5700 years ago in the Mongolian Plateau in China [2]. China has various sheep resources, including 42 indigenous sheep breeds [3]. These breeds are well adapted to the local plateau and desert environments [4], but the meat yield of these Chinese indigenous sheep breeds is so poor that it fails to meet the increasing consumer demand for mutton.

From the 1960s, in Bayannur of the Inner Mongolia Autonomous Region, German Mutton Merino sheep were imported and bred with indigenous Mongolian sheep to improve their meat yield. After 40 years of selection and improvement, in 2007, a novel breed, Bamei mutton sheep, was created and showed good genetic stability [5]. Under grazing conditions, Bamei mutton sheep is well adapted to dry and chilly winters in the Inner Mongolia Autonomous Region. Approximately 57,000 sheep of this type are raised mainly in this region. The lambs of Bamei mutton sheep grow faster than those of Small-Tail Han sheep. In terms of the live weight, they can reach about $53 \mathrm{~kg}$ at the age of 8 months and their average daily gain was found to be $199.54 \mathrm{~g}$ from 4 to 8 months of age under intensive feeding patterns [6]. Bamei mutton sheep are an important male breed in commercial hybrid sheep production in Bayannur [7]. The meat trait performance of this breed is excellent, but there is large variation in its reproductive abilities. The average lambing rate of the Bamei mutton nucleus group was over 150\% [8]. The maintenance of high levels of fertility is vital for efficient sheep production [9]. Therefore, improving the level of fecundity of this new breed is a major focus of breeders.

Litter size has a major impact on desirable economic traits of sheep (e.g., meat, wool, and milk). Increasing litter size can improve the efficiency of sheep production [9]. The main factors affecting sheep fecundity include ovulation, uterine capacity, and placental efficiency. Litter size is a complex trait, but some major genes affecting prolificacy have been discovered in recent years. The $F e c B$ (bone morphogenetic protein $1 \mathrm{~B}$ receptor, BMPR1B) gene was the first major gene found to affect prolificacy. Subsequently, many major genes, such as $\mathrm{FeCX}$ (bone morphogenetic protein 15, BMP15), FecG (growth differentiation factor 9, GDF9), FecL (glycosylation enzyme beta-1,4-N-acetyl-galactosaminyltransferase 2, B4GALNT2), and LEPR (leptin receptor), were also reported [10,11].

As reported in Science, the first high-quality $2.61 \mathrm{~Gb}$ reference genomes of domestic sheep were sequenced and assembled in 2014. They can help in identifying genomic signatures of domestic traits in sheep [12]. By performing whole-genome resequencing on phenotypically divergent sheep populations, some selective sweeps were identified relating to important traits targeted by artificial selection during domestication, such as horn morphology, [1,13], coat color [14], tail morphology and fat deposition $[3,15,16]$ and variation of thoracic vertebrae [17].

Regarding reproductive traits, using selection tests in pigs, previous studies demonstrated some strong selective signals belonging to the TGF- $\beta$ signaling pathway $[18,19]$. In addition, in goats, the genes regulating seasonal reproduction and litter size have been specifically selected $[20,21]$. Moreover, some fecundity-related genes revealed a strong selection signature in sheep from Ethiopia and Europe [22,23].

Against this background, we performed whole-genome resequencing of 10 ewes selected to have extreme fecundity and sweeping analysis to identify the underlying variants and genes responsible for the litter size of Bamei mutton sheep under the influence of artificial selection.

\section{Materials and Methods}

\subsection{Animals and DNA Preparation}

In this study, we collected whole-blood samples from the jugular vein of 10 Bamei mutton sheep at the Xianghe Bamei mutton sheep-breeding park (Bayannur, China). These 
blood samples were placed in EDTA vacutainer tubes for storage. These ewes were about 3 years old and selected from among 500 sheep. Only ewes with litter size data showing that they had given birth more than three times were sampled. The selected ewes were grouped into two categories based on the phenotype of litter size (monotocous sheep giving birth to only one lamb in three consecutive parities and polytocous sheep giving birth to more than two lambs in two consecutive parities). Then, genomic DNA was extracted from $200 \mu \mathrm{L}$ of sheep blood using a QIAamp DNA Blood Mini Kit (Qiagen, Hilden, Germany), in accordance with the manufacturer's instructions. DNA quality and integrity were assessed by spectrophotometry (OD260/280) and 1.0\% gel electrophoresis.

\subsection{Genome Sequencing}

High-quality DNA for Illumina sequencing library construction was randomly sheared into small pieces (300-400 bp). After end-repair, "A"-tailing and ligating to Illumina sequencing adapters, $400-500 \mathrm{bp}$ ligated products were amplified by ligation-mediated PCR (LM-PCR). Then, $2 \times 100$ bp paired-end sequencing was carried out on an Illumina HiSeq 2500 sequencer and the original data were analyzed by Illumina HiSeq Control Software (Illumina, San Diego, CA, USA).

\subsection{Read Processing and Variant Calling}

NGS QC Toolkit v2.3.3 was used for quality control of the raw reads following three steps [24]. First, reads with $>70 \%$ low-quality bases (score $<20$ ) in the FASTQ files were filtered out. Second, reads containing $\mathrm{N}$ residues were filtered out. Third, low-quality ends (scores $<20$ ) were trimmed. After this trimming, if the read length was $<35$, the read was removed. After this quality control, the reads of each sheep were mapped to the sheep genome assembly v3.1 (ftp:/ /ftp.ncbi.nlm.nih.gov / genomes/all/GCA_00029873 5.1_Oar_v3.1/GCA_000298735.1_Oar_v3.1_genomic.fna.gz) using BWA v0.6.2 [25]. The bam file was sorted by chromosome and duplicated reads were removed using SAMtools v0.1.19 [26].

Mapped reads of all samples were pooled for variant calling using SAMtools, with the parameters "mpileup-u-C50-DS-q20." The .vcf file was generated by bcftools view with the parameter "-evcgN." Then, vcfutils.pl with minimum depth "-d 20" and maximum depth "-D 300" was used to filter raw variants. Finally, the variants were annotated by ANNOVAR [27] version 2014-11-12, in accordance with Ensembl gene annotation (Oar_v3.1).

\subsection{Population Genetics Analysis}

The samples were separated into two groups (five monotocous individuals, five polytocous individuals). The SNP densities, minor allele frequencies, and Tajima's D of each group were calculated by VCFtools v0.1.12b (https:/ /vcftools.github.io/index.html) [28]. Only SNPs in autosomes were preserved for the phylogenetic analysis. A phylogenetic tree of all samples was generated using SNPhylo [29] version 20160204 (http:/ / chibba.pgml.uga. edu/snphylo/), based on the maximum likelihood method. A total of 500,000 SNPs were randomly selected for calculating the linkage disequilibrium (LD) $\mathrm{r}^{2}$ using Haploview [30] with parameters set as follows: "-missingCutoff 0.2 -dprime -minMAF 0.1." The SNP pairs were clustered based on the physical distances of these genes. The average LD (e.g., $0-1 \mathrm{~kb}$ ) of each group was represented by the mean $\mathrm{r}^{2}$.

\subsection{Selective Sweep Analysis}

The pooled heterozygosity Hp was calculated over $10 \mathrm{~kb}$ windows using the formula:

$$
H p=2 \times \frac{\sum n_{M A J} \times \sum n_{M I N}}{\left(\sum n_{M A J}+\sum n_{M I N}\right)^{2}}
$$

where $\sum n_{M A J}$ denotes the sum of major allele frequencies in a selected window and $\sum n_{\text {MIN }}$ denotes the sum of minor allele frequencies [31]. 
$\mathrm{F}_{\mathrm{ST}}$ values were calculated between monotocous individuals and polytocous individuals for single SNPs using a method that adjusts for a small sample size [32]. We averaged $\mathrm{F}_{\mathrm{ST}}$ values over $50 \mathrm{~kb}$ sliding windows along the genome with the Bio::PopGen::PopStats Package in BioPerl [33] and Z-transformed the resultant distribution. Putative selection targets were extracted from the extreme tail of the distribution by applying a $\mathrm{Z}\left(\mathrm{F}_{\mathrm{ST}}\right)>5$ cut-off [34].

fastPHASE v1.4.0 was used to phase the genotypes of all samples with the parameters "-T10-K8" [35]. Then, the phased data were used to calculate the cross-population extended haplotype homozygosity (XP-EHH) value by XP-EHH [36]. XP-EHH values were averaged over $50 \mathrm{~kb}$ sliding windows. These scores approximately followed a normal distribution; the threshold to locate putatively selected regions was two times the XP-EHH distribution standard deviation ( $|\mathrm{XP}-\mathrm{EHH}|>2$ ). Manhattan plots for $\mathrm{F}_{\mathrm{ST}}$ and XP-EHH were generated with the R package gap [37].

A phylogenetic tree was generated using all variants located in these regions. Candidate genes targeted by positive selection were defined as genes overlapping with sweep regions $\left(\mathrm{ZF}_{\mathrm{ST}}>5\right.$ and $\left.|\mathrm{XP}-\mathrm{EHH}|>2\right)$. GO (gene ontology) and KEGG (kyoto encyclopedia of genes and genomes) enrichment analyses for candidate genes were performed by DAVID 6.8 [38], and $p$ values were corrected using the Benjamini-Hochberg method. Protein-altering mutations in these genes were listed and ranked by their single-site $\mathrm{F}_{\mathrm{ST}}$ value. The protein-altering mutations of the candidate sweep gene $K D M 4 B$ were localized to regions that are evolutionarily conserved among mammalian species.

\subsection{Sanger Sequencing Validation}

To confirm the SNPs detected in exons of the genes selected by sweep analysis, we selected eight SNPs from six genes and designed primers for their Sanger sequencing (Supplementary Table S1). Then, we outsourced the amplification and screening of SNPs in 15 monotocous and 14 polytocous sheep of this group to GENENODE (Wuhan, China).

\section{Results}

\subsection{Sequencing and Mapping of the Sheep}

We sequenced five monotocous and five polytocous sheep using an Illumina HiSeq2500 sequencer, generating a total of 627.16 million raw read pairs, comprising $180.16 \mathrm{~Gb}$ of raw data. After filtering out the low-quality reads, each sheep was mapped to the sheep reference genome, with an average alignment rate of $93.76 \%(92.79-94.34 \%)$. The percentages of reads sequenced at least once per bp varied from $87.38 \%$ to $94.55 \%$. The percentage of reads sequenced at least four times per bp was $>61.82 \%$ (Supplementary Table S3).

\subsection{Identification of the Variation of the Sheep}

We obtained about 21.39 million SNPs and 2.04 million insertions and deletions (indels) for all sheep in the two groups. Most SNPs $(\sim 71.7 \%)$ were located within intergenic regions, while only a few $(\sim 0.6 \%)$ were located within coding regions. The exonic SNPs were identified (Supplementary Table S4). There were 45,775 vs. 48,265 SNPs involving nonsynonymous mutations, 742 vs. 785 involving stop-gain variation, and 45 vs. 52 involving stop-loss nonsense variation in the polytocous and monotocous sheep, respectively. The rates of heterozygosity of the polytocous and monotocous groups were $30.02 \%$ and $30.07 \%$, respectively. The transition-to-transversion ratios (ts/tv) were almost identical between the two groups (2.4346 for the polytocous population versus 2.4352 for the monotocous population) (Supplementary Table S5).

\subsection{Population Structure Analysis}

The levels of genome-wide genetic diversity, Tajima's D, and the minor allele frequency (MAF) distribution indicated that high-frequency minor alleles constitute a small proportion of the total but are slightly more abundant in polytocous sheep than in monotocous ones (Supplemental Figure S1). Additionally, haplotype analysis indicated that 
the polytocous sheep have slower decay of pairwise correlation coefficient $\left(\mathrm{r}^{2}\right)$ and higher integrated haplotype homozygosity $(\mathrm{iHH})$ than the monotocous sheep (Supplemental Figure S2).

To determine the phylogenetic relationship between monotocous and polytocous sheep, a neighbor-joining tree was constructed using high-quality SNPs. When the tree was generated using the SNPs for the whole genome, monotocous and polytocous sheep formed a mixed clade (Figure 1A). This indicated that the pairwise distances within each group were larger than those between the groups and there was no significant distinction between the two groups. However, in the phylogenetic tree constructed using SNP data selected based on $\mathrm{F}_{\mathrm{ST}}$ score, the monotocous and polytocous sheep were classified into two genetically different groups (Figure 1B). The results of the two trees show that the population genetic structure was not associated with the litter size and that the breeding time of high-fecundity Bamei mutton sheep was relatively short.

A

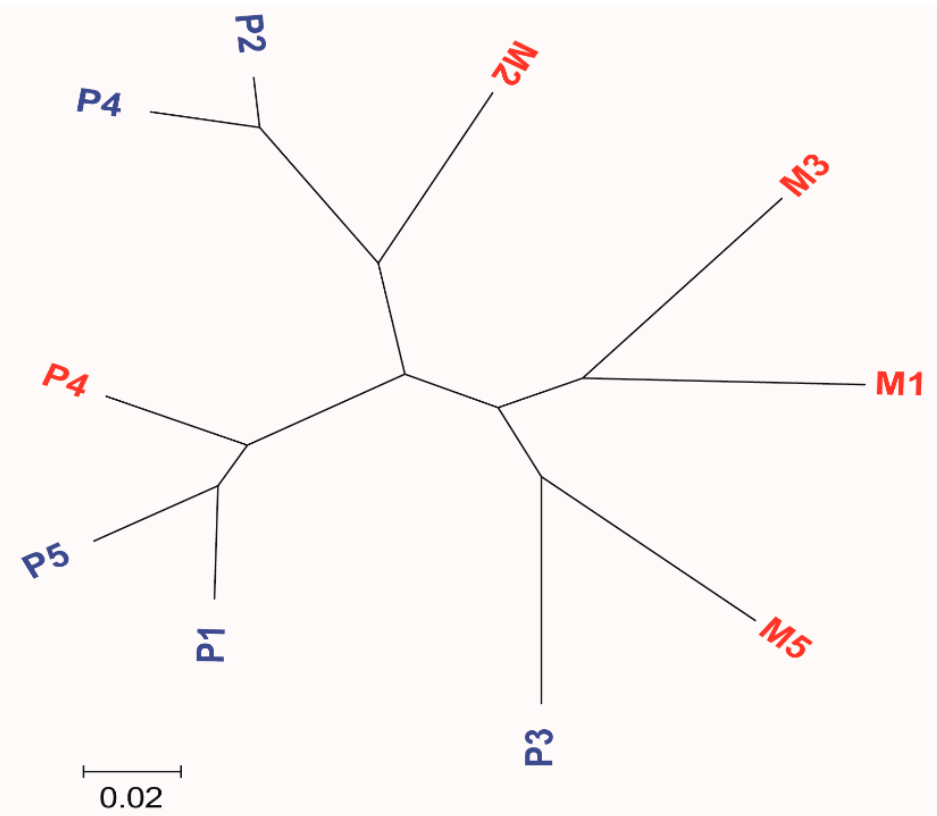

B

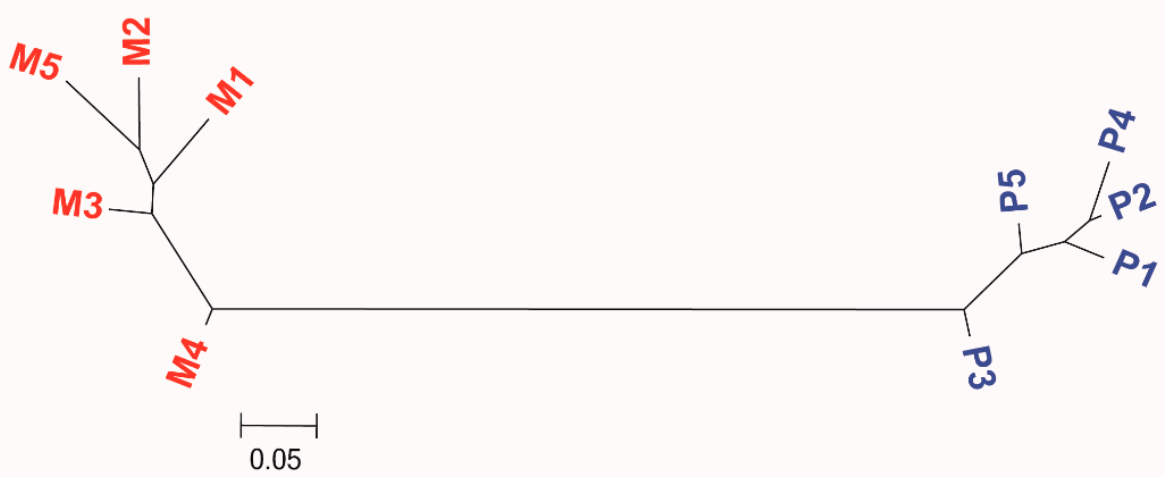

Figure 1. Phylogenetic and population structure of monotocous and polytocous sheep. (A) A neighbor-joining phylogenetic tree constructed using whole-genome SNP data. The scale bar represents the level of similarity; Monotocous (red) and polytocous (blue) samples are indicated. (B) A neighbor-joining phylogenetic tree constructed using SNP data selected based on $\mathrm{F}_{\mathrm{ST}}$ score.

\subsection{Analysis of the Selected Loci and Candidate Genes}

To find selection signals associated with high prolificacy, the average $\mathrm{F}_{\mathrm{ST}}$ values were calculated for nonoverlapping $50 \mathrm{~kb}$ windows on the autosomes and $X$ chromosome. After $\mathrm{Z}$-transforming the values, we selected the windows with $\mathrm{Z}\left(\mathrm{F}_{\mathrm{ST}}\right)>5$ across the genome, as 
in previous studies [34]. In total, we identified 85 unique autosomal regions and two Xchromosome regions containing 81 candidate genes. The region with the strongest selective signal $\left(\mathrm{F}_{\mathrm{ST}}=0.6639187, \mathrm{ZF}_{\mathrm{ST}}=10.65708746\right)$ between the monotocous and polytocous sheep was located on chromosome 5 (16750000-16800000 bp), which contains KDM4B (lysine demethylase 4B) (Figure 2A, Supplementary Table S6).

A

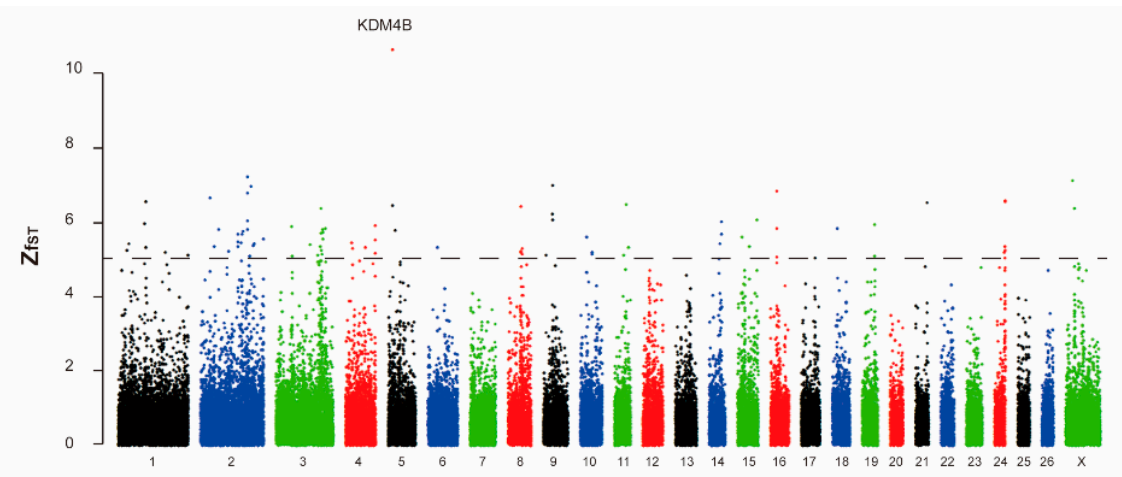

Chromosomes

B

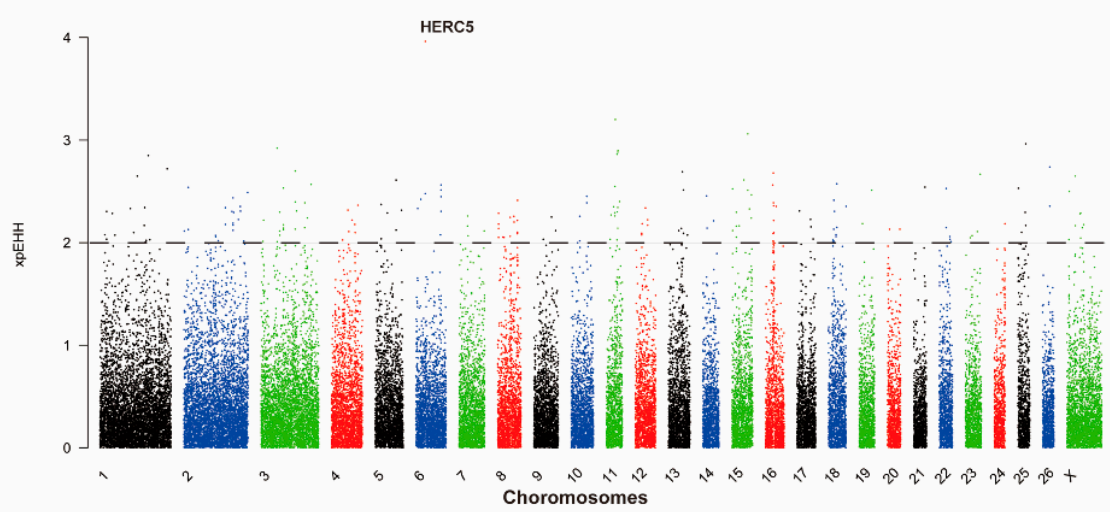

Figure 2. Selective sweep analysis of the monotocous and polytocous sheep. (A) Manhattan plot of FST among the monotocous and polytocous sheep. The FST was calculated for each $50 \mathrm{~kb}$ autosomal and X-chromosome window. The dashed line denotes a threshold of $Z(F S T)=5 ;(B)$ Manhattan plot of $\mathrm{XP}-\mathrm{EHH}$ among the monotocous and polytocous sheep. The XP-EHH value was calculated for each $50 \mathrm{~kb}$ autosomal and X-chromosome window. The dashed line denotes a threshold of $|\mathrm{XP}-\mathrm{EHH}|>2$.

We also estimated the XP-EHH statistic for the monotocous and polytocous groups using monotocous sheep as a control. An XP-EHH value greater than zero indicates that these sites have been selected in the monotocous population, while a value less than zero indicates that selection has occurred in the polytocous population. We scanned the regions using the threshold $|\mathrm{XP}-\mathrm{EHH}|>2$ as candidate regions. A total of 198 regions including 162 genes were found to have undergone positive selection in the XP-EHH analysis, with 155 regions having undergone selection in the polytocous population. A region of chromosome $6(36,200,000-36,250,000 \mathrm{bp})$ associated with strong selection was found to have the largest | XP-EHH | value (XP-EHH = -3.963) (Figure 2B, Supplementary Table S7).

We combined the genes obtained by the two methods described above. A total of 221 genes were found to have been selected in total. Overall, 14 genes were positively selected across both of the two methods (Supplementary Table S8).

Gene Ontology and KEGG pathway analyses were performed to further study the functions of the selected genes identified by the two methods. The enriched GO terms ( $p$-value $<0.1$ ) and KEGG pathways $(p$-value $<0.5)$ are shown in Supplementary Tables S9 and S10. By the Functional Annotation Clustering tool of DAVID 6.8, we identified two annotation clusters (classification stringency: Medium). Annotation cluster 2 contained the GnRH signaling pathway, estrogen signaling pathway, and oxytocin signaling pathway. The reproductive hormones in these pathways are involved in regulating sheep estrus, 
follicle development, and ovulation. JUN (JUN proto-oncogene, AP-1 transcription factor subunit), ITPR3 (inositol 1,4,5-trisphosphate receptor type 3), and PLCB2 (phospholipase C beta 2) were enriched in all three pathways (Table 1).

Table 1. The functional annotation clusters of similar biological meanings sharing common gene members enriched by DAVID.

\begin{tabular}{|c|c|c|c|c|}
\hline Annotation Cluster 1 & Enrichment Score: 1.95 & & & \\
\hline Category & Term & Count & Genes & $p$-Value \\
\hline KEGG_PATHWAY & $\begin{array}{l}\text { oas04660:T cell receptor } \\
\text { signaling pathway }\end{array}$ & 8 & $\begin{array}{c}\text { CD3D CD3E } \\
\text { CD3G JUN } \\
\text { MALT1 CARD11 } \\
\text { MAP3K14 PAK6 }\end{array}$ & $1.35 \times 10^{-5}$ \\
\hline KEGG_PATHWAY & $\begin{array}{c}\text { oas05142:Chagas disease } \\
\text { (American } \\
\text { trypanosomiasis) }\end{array}$ & 5 & $\begin{array}{c}\text { CD3D CD3E } \\
\text { CD3G JUN } \\
\text { PLCB2 }\end{array}$ & 0.013 \\
\hline KEGG_PATHWAY & oas05166:HTLV-I infection & 6 & $\begin{array}{c}\text { ATM CD3D } \\
\text { CD3E CD3G } \\
\text { JUN MAP3K14 }\end{array}$ & 0.072 \\
\hline KEGG_PATHWAY & oas05162:Measles & 4 & $\begin{array}{l}\text { CD3D CD3E } \\
\text { CD3G ADAR }\end{array}$ & 0.094 \\
\hline KEGG_PATHWAY & $\begin{array}{l}\text { oas04640:Hematopoietic } \\
\text { cell lineage }\end{array}$ & 3 & $\begin{array}{l}\text { CD3D CD3E } \\
\text { CD3G }\end{array}$ & 0.157 \\
\hline Annotation Cluster 2 & Enrichment Score: 0.67 & & & \\
\hline Category & Term & Count & Genes & $p$-Value \\
\hline KEGG_PATHWAY & $\begin{array}{c}\text { oas04912:GnRH signaling } \\
\text { pathway }\end{array}$ & 3 & $\begin{array}{l}\text { JUN ITPR3 } \\
\text { PLCB2 }\end{array}$ & 0.151 \\
\hline KEGG_PATHWAY & $\begin{array}{l}\text { oas04915:Estrogen } \\
\text { signaling pathway }\end{array}$ & 3 & $\begin{array}{l}\text { JUN ITPR3 } \\
\text { PLCB2 }\end{array}$ & 0.188 \\
\hline KEGG_PATHWAY & $\begin{array}{l}\text { oas04921:Oxytocin } \\
\text { signaling pathway }\end{array}$ & 3 & $\begin{array}{l}\text { JUN ITPR3 } \\
\text { PLCB2 }\end{array}$ & 0.340 \\
\hline
\end{tabular}

\subsection{Mutations in the KDM4B Gene}

$K D M 4 B$ is located in the highly differentiated region with the highest $\mathrm{F}_{\mathrm{ST}}$ value between the monotocous and polytocous groups. A selected mutation that goes to fixation tends to reduce variation in linked sites in the process of a selective sweep [39]. Therefore, we determined the $\mathrm{Hp}$ value of the window (chr 5: 16,750,000-16,800,000 bp) around $K D M 4 B$. The $\mathrm{Hp}$ value of the polytocous group $(\mathrm{Hp}=0.0818)$ decreased in the KDM4B region and was the lower than in the monotocous group ( $\mathrm{Hp}=0.22923)$ (Figure 3A).

To identify SNVs subjected to selection, we screened the exonic mutations of the $K D M 4 B$ gene in both monotocous and polytocous groups. SNVs that can alter protein translation, structure, and even function may contribute to rapid evolution in domestic animals [40]. Here, 13 synonymous SNVs, 3 nonsynonymous SNVs, and 1 frameshift deletion were identified. All of the three nonsynonymous SNVs cause amino acid sequence changes p.S570G $\left(\mathrm{F}_{\mathrm{ST}}=0.11\right)$, p.S924L $\left(\mathrm{F}_{\mathrm{ST}}=0\right)$, and p.S936A $\left(\mathrm{F}_{\mathrm{ST}}=0.45\right)$ in the translated protein (in accordance with Ensembl gene annotation) (Supplementary Table S11).

We compared the $\mathrm{F}_{\mathrm{ST}}$ values of the three nonsynonymous SNVs; only mutation p.S936A had high divergence of allele frequency within our population: monotocous sheep $50 \%(n=5)$ and polytocous sheep $90 \%(n=5)$. To confirm these frequencies based on Illumina sequencing, alleles of additional samples were genotyped by Sanger sequencing. The frequency of mutant allele of $K D M 4 B$ gene genotyped by Sanger sequencing showed a slight decrease in polytocous group $60 \%(n=14)$, compared with the result in whole genome sequencing. The mean of individual litters variants in polytocous sheep may affect the distribution of the genotype when increasing number of samples. The frequency of the mutant homozygote in polytocous sheep was higher than that in monotocous sheep. Moreover, the distribution of KDM4B p.S936A genotype contained significant differeces 
between polytocous and monotocous sheep population genotyped by Sanger sequencing $(p$-value $=0.041)$. (Figure 3B). This indicates that the nonsynonymous mutations may be associated with the selective sweep at KDM4B.

A
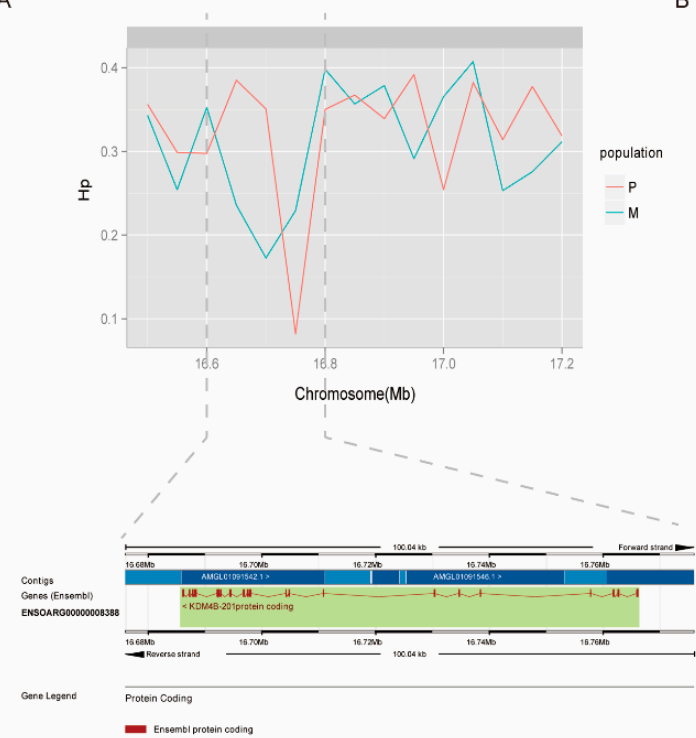

C

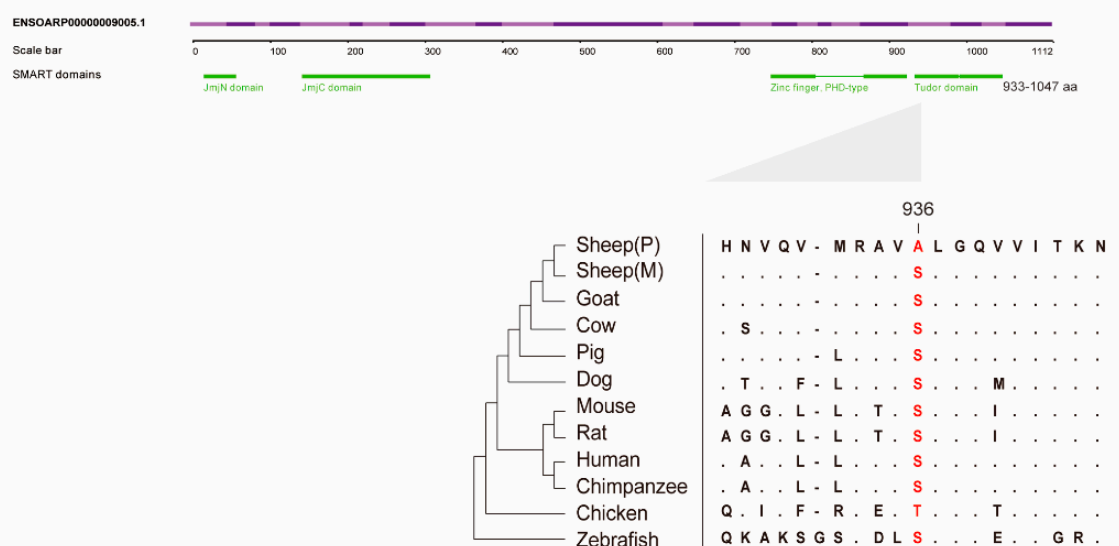

B

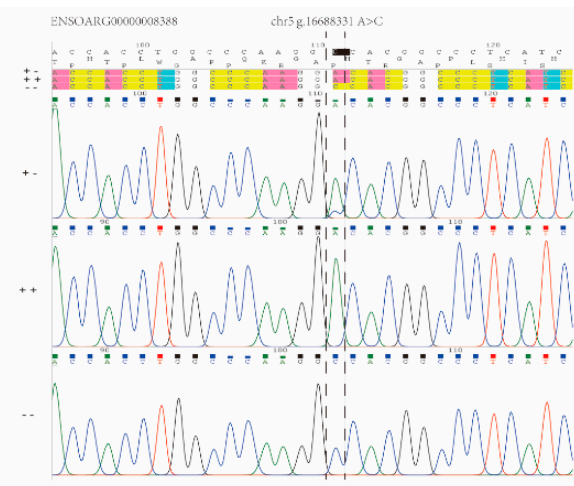

exon21:p.S936A

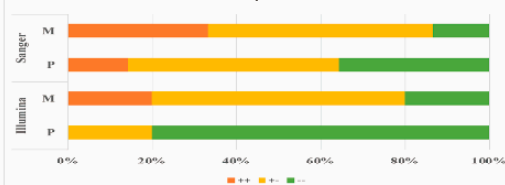

Figure 3. KDM4B mutation in the coding region. (A) Hp value around the KDM4B loci. Hp was calculated for each $10 \mathrm{~kb}$ window of the monotocous and polytocous sheep. The gene coordinates are based on Ensembl ID ENSOARG00000008388; (B) Percentages of homozygotes and heterozygotes in the monotocous and polytocous sheep. The reference and mutant alleles are represented by "+" and "-," respectively. Besides the 10 sheep sequenced with Illumina technology, additional samples were genotyped by Sanger sequencing; (C) Evolutionary analysis of the p.S936A amino acid variant. The protein coordinates are based on Ensembl ID ENSOARP00000009005.1. The upper panel shows the domains of the protein. The orthologous protein sequences from 11 vertebrates are aligned with the mutant residues shown in red. The NJ tree derived from the multiple alignment is shown in the lower panel.

p.S936A affected the TUDOR domain (Figure 3C). This domain can bind to specific lysine methylation marks on histone proteins (H3-K4me3, H3-K23me3, and H4-K20me3). It plays a vital role in chromatin localization and the regulation of enzymatic function [41]. Thus, we aligned the KDM4B protein mutant with its ortholog in diverse vertebrates to evaluate the functional effects of the variants. The results reveal that p.S936A is quite well conserved, being invariant among all of the other mammals that we used (Figure 3C). All of the results indicate that p.S936A is an important mutation for the reproduction-related KDM4B sweep. 


\section{Discussion}

Continuous artificial selection in production-oriented breeding has left selective signatures and genomic variability in domesticated sheep. In this study, we performed whole-genome sequencing of 10 Bamei mutton sheep with different litter sizes from the same population. In this population, reproductive traits have undergone intensive selection via the breeding strategy. In this research, numerous mutations were selected in the population after screening, but the rates of heterozygosity did not differ between the two populations. When a neighbor-joining tree was constructed using SNPs selected based on

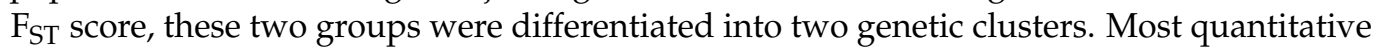
traits are known to respond quickly to artificial selection, and this population subjected to selection of litter size might have evolved in opposite directions with soft sweeps [42,43].

Selective sweeps can identify important genomic regions that have been swept in the recent past. Some genes associated with complex, economically important traits have been identified with the help of selected sweep analysis by whole-genome sequencing. We used the XP-EHH test to detect alleles near fixation within a Bamei mutton sheep population. Upon undergoing recent selection, the selected allele generally reaches a high frequency or fixation in one group, but remains polymorphic in the whole population [44]. In this research, we discovered 155 regions selected for in the polytocous population, which was greater than the 43 regions screened in the monotocous population. Using fixed window selection of $\mathrm{F}_{\mathrm{ST}}$ and $\mathrm{XP}-\mathrm{EHH}, 221$ candidate genes were found to be associated with the prolificacy trait. In a previous study using a segregated flock based on QTL and GWAS mapping, some mutations ( $\mathrm{FecB}, \mathrm{FecX}$, and $\mathrm{FecG}$ ) were identified to affect ovulation in sheep [11]. However, here we did not identify any mutations previously reported to increase the number of ovulations in sheep. The litter size per breeding ewe is not only influenced by ovulation, but also affected by a number of factors including fertilization rate and pregnancy loss (in the embryonic and fetal development period). Recently, the LEPR gene, estrogen receptor 1 (ESR1) gene, and prolactin (PRL) gene were found to be associated with fecundity, as revealed by a selective sweep analysis in European commercial and semi-feral breeds and a Chinese indigenous breed distributed in different ecoregions $[23,45,46]$.

JUN, ITPR3, and PLCB2 in the selected region were enriched in gonadotrophin releasing hormone $(\mathrm{GnRH})$, oxytocin, and estrogen signaling pathway associated with the complex process from estrus to lambing. This process is organized by complex communication among the hypothalamus, pituitary, ovary, and uterus. GnRH, secreted by the hypothalamus, regulates the synthesis and release of gonadotrophins, follicle-stimulating hormone (FSH), and luteinizing hormone (LH) from the pituitary [47]. FSH and LH support the growth and maturation of follicles. Estradiol and progesterone secreted by the corpus luteum inhibit GnRH release by feedback modulation. During the follicular phase, following luteolysis, estradiol reaches a critical threshold and stimulates the preovulatory gonadotrophin surge, appearing to help the ovulation of mature follicles [11].

PLCB is involved in a wide range of signals of reproductive processes, being regulated by many hormones (FSH, LH, GnRH, oxytocin). PLCB2 as a member of the PLCB subfamily is activated by G-protein-linked receptors and can hydrolyze phosphatidylinositol 4,5bisphosphate to form inositol-1,3,4-trisphosphate (IP3) and diacylglycerol (DAG), which stimulate $\mathrm{Ca}^{2+}$ release and protein kinase $\mathrm{C}$ activity, respectively [48]. ITPR3 is a member of the IP3 receptor family. IP3, as an intracellular secondary messenger, mobilizes $\mathrm{Ca}^{2+}$ from endoplasmic reticulum stores, which transduces several calcium-dependent cascades. IP3 receptor downregulation induced by GnRH can suppress the secretion of LH/FSH [49,50].

JUN is one of three JUN members [JUN (c-JUN), JUNB, and JUND] constituting the AP-1 family of heterodimeric transcription factors by combining with four FOS members [FOS (c-Fos), FOSB, FRA1, and FRA2]. GnRH can stimulate the expression of JUN by rapidly and transiently binding to the AP1 site in the FSHB promoter and then stimulating FSHB transcription [50]. Conditional knockout of JUN in mice resulted in subfertility 
in both sexes, such as impaired spermatogenesis in males, diminished corpora lutea in females, and lower gonadal steroid (GnRH and LH) levels [51].

The nonsynonymous mutation p.S936A at KDM4B was discovered and confirmed in monotocous and polytocous sheep; it may be the reason for the signal of a selective sweep at the $K D M 4 B$ locus. $K D M 4 B$ belonging to the KDM4/JMJD2 family of histone demethylases contains a JmjN domain, JmjC domain, tandem plant homeodomains (PHD), and tandem Tudor domains. KDM4B catalyzes the demethylation of $\mathrm{H} 3 \mathrm{~K} 9 \mathrm{me} 3$ and $\mathrm{H} 3 \mathrm{~K} 9 \mathrm{me} 2$ at or near regulated promoters to promote expression of the downstream pathway induced by multiple different extracellular stimuli [52]. KDM4B plays a central role in regulating the Estrogen Receptor (ER) signaling cascade by controlling expression of the ER and FOXA1 genes. These two important genes can maintain the estrogen-dependent phenotype [53]. In the developing ovarian follicle, granulosa cells are the main producers of estrogen. $K D M 4 B$ is expressed in granulosa cells at early stages of folliculogenesis and its level is correlated with pregnancy failure in IVF patients [54]. KDM4B expression in the uterus is also associated with recurrent pregnancy loss in women [55]. The identified intersection between steroid hormones and KDM4B in the ovary and uterus sheds new light on the regulation of reproduction.

\section{Conclusions}

Following high-throughput sequencing, SNPs and indels were identified from two sheep populations. The sequencing data revealed the genetic diversity and population differentiation of the Bamei mutton sheep population experiencing selection for litter size. The genomic selection scan detected some interesting candidate genes and pathways under artificial selection, which might have increased litter size. This genome-wide research provides valuable information for future whole-genome selection for fecundity in this sheep breed.

Supplementary Materials: The following are available online at https://www.mdpi.com/2076-2 615/11/1/157/s1, Figure S1: Distributions of (A) minor allele frequency and (B) Tajima's D across the genome of Monotocous (Blue) and polytocous (Red), Figure S2: Levels of linkage disequilibrium across the genome of Monotocous (Blue) and polytocous (Red), Table S1: Primer information of Sanger sequencing, Table S2: Sample information and sequencing quality, Table S3: Reads mapping statistics and coverage of depth for Monotocous and Polytocous sheep, Table S4: Number of SNPs and Indels in different chromosomes, Table S5: Number of annotated SNPs in different gene regions, Table S6: Genomic regions and genes highly differentiated (Z(FST) > 5) between Monotocous and Polytocous sheep, Table S7: Candidate regions and genes of 50-kb windows with value of IXPEHH | $>2$ in Monotocous and Polytocous sheep, Table S8: Overlapping selected windows selected by FST and XP-EHH, Table S9: Gene Ontology enrichment analysis of the 221 candidate genes with GO terms $(p<0.1)$, Table S10: Ranking of KEGG pathway with Pvalue $(p<0.5)$ enriched from 221 genes, Table S11: Mutations in exons of KDM4B gene.

Author Contributions: X.W., Z.P., and M.C. conceived the study. Z.P., X.G., and X.H. contributed to sample collection. X.W., Y.Y., and Q.L. analyzed the data. X.W. and Y.Y. wrote the manuscript. X.W., Y.Y., R.D., Q.L., W.H., S.G., and M.C. revised the manuscript. All authors have read and agreed to the published version of the manuscript.

Funding: This work was supported by The Genetically Modified Organisms Breeding Major Program of China (2016ZX08009-003-006), by the Major Science and Technology Program of Inner Mongolia Autonomous Region of China, by the National Natural Science Foundation of China (No. 31501926, No. 31772580, No. 31572371, No. 31861143012), by the Central Public-interest Scientific Institution Basal Research Fund (No. Y2017JC24, No. 2015ywf-zd-8, No. 2018-ywf-yb-2), by The Agricultural Science and Technology Innovation Program of China (No. ASTIP-IAS13), by The Earmarked Fund for China Agriculture Research System (No. CARS-38), by The Youth Innovative Research and Experimental Project of Tianjin Academy of Agricultural Sciences (No. 201915), and by Joint Funds of The National Natural Science Foundation of China and The Government of Xinjiang Uygur Autonomous Region of China (No. U1130302). 
Institutional Review Board Statement: The study was conducted according to the guidelines of the Animal Management Committee (in charge of animal welfare issue) of the Institute of Animal Science, Chinese Academy of Agricultural Sciences (IAS20160322; IAS-CAAS, Beijing, China).

Informed Consent Statement: Not applicable.

Data Availability Statement: The raw genomic sequencing data from this work have been uploaded to the NCBI SRA database with accession number PRJNA560632 (https:/ / www.ncbi.nlm. nih.gov/sra/PRJNA560632). The variation data reported in this paper has been deposited in the Genome Variation Map [56] in National Genomics Data Center [57], China National Center for Bioinformation/Beijing Institute of Genomics, Chinese Academy of Sciences, under accession number GVM000114 at http:/ /bigd.big.ac.cn/gvm/getProjectDetail?project=GVM000114.

Acknowledgments: We thank Liwen Bianji, Edanz Group China (www.liwenbianji.cn/ac), for editing the English text of a draft of this manuscript.

Conflicts of Interest: The authors declare no conflict of interest.

\section{References}

1. Kijas, J.W.; Lenstra, J.A.; Hayes, B.; Boitard, S.; Porto Neto, L.R.; San Cristobal, M.; Servin, B.; McCulloch, R.; Whan, V.; Gietzen, K.; et al. Genome-Wide Analysis of the World's Sheep Breeds Reveals High Levels of Historic Mixture and Strong Recent Selection. PLoS Biol. 2012, 10, e1001258. [CrossRef] [PubMed]

2. Zhao, Y.-X.; Yang, J.; Lv, F.-H.; Hu, X.-J.; Xie, X.-L.; Zhang, M.; Li, W.-R.; Liu, M.-J.; Wang, Y.-T.; Li, J.-Q.; et al. Genomic Reconstruction of the History of Native Sheep Reveals the Peopling Patterns of Nomads and the Expansion of Early Pastoralism in East Asia. Mol. Biol. Evol. 2017, 34, 2380-2395. [CrossRef] [PubMed]

3. Wei, C.; Wang, H.; Liu, G.; Wu, M.; Cao, J.; Liu, Z.; Liu, R.; Zhao, F.; Zhang, L.; Lu, J.; et al. Genome-wide analysis reveals population structure and selection in Chinese indigenous sheep breeds. BMC Genom. 2015, 16, 194. [CrossRef] [PubMed]

4. Yang, J.; Li, W.-R.; Lv, F.-H.; He, S.-G.; Tian, S.-L.; Peng, W.-F.; Sun, Y.-W.; Zhao, Y.-X.; Tu, X.-L.; Zhang, M.; et al. Whole-Genome Sequencing of Native Sheep Provides Insights into Rapid Adaptations to Extreme Environments. Mol. Biol. Evol. 2016, 33, 2576-2592. [CrossRef]

5. Jin, X.; Su, R.; Zhang, W.; Li, J. The identification and assessment on genetic characteristics in grading breeding sheep populations with microsatellite markers. Agric. Sci. Technol. 2008, 9, 21-24.

6. Zhao, T.; Zhang, H.; Wang, W.; Chen, H.; Jiang, H.; Ma, Y.; Luo, H. Comparison of meat-productivities between Bamei Sheep and Small-tall Han Sheep under intensive feeding pattern. J. China Agric. Univ. 2014, 19, 121-128.

7. Zhang, H.; Liu, S.; Jin, Y.; Jin, Z.; Yuan, Q.; Jia, X. Study on growth and meat output in different carcass levels of Bamei mutton sheep and their hybrid progeny. Meat Ind. 2013, 2013, 17-20.

8. Tan, X.; Li, B.; Zhao, Y. Current Status and Developing Prospect of Sheep and Goat Breeding in China. Chin. J. Anim. Sci. 2015, 51, 15-19.

9. Notter, D.R. Genetic aspects of reproduction in sheep. Reprod. Domest. Anim. 2008, 43 (Suppl. 2), 122-128. [CrossRef]

10. Liu, Q.; Pan, Z.; Wang, X.; Hu, W.; Di, R.; Yao, Y.; Chu, M. Progress on major genes for high fecundity in ewes. Front. Agric. Sci. Eng. 2014, 1, 282-290. [CrossRef]

11. Juengel, J.L. How the quest to improve sheep reproduction provided insight into oocyte control of follicular development. J. $R$. Soc. N. Z. 2018, 1-21. [CrossRef]

12. Jiang, Y.; Xie, M.; Chen, W.; Talbot, R.; Maddox, J.F.; Faraut, T.; Wu, C.; Muzny, D.M.; Li, Y.; Zhang, W.; et al. The sheep genome illuminates biology of the rumen and lipid metabolism. Science 2014, 344, 1168-1173. [CrossRef]

13. Pan, Z.; Li, S.; Liu, Q.; Wang, Z.; Zhou, Z.; Di, R.; Miao, B.; Hu, W.; Wang, X.; Hu, X.; et al. Whole-genome sequences of 89 Chinese sheep suggest role of RXFP2 in the development of unique horn phenotype as response to semi-feralization. GigaScience 2018, 7. [CrossRef]

14. Fariello, M.I.; Servin, B.; Tosser-Klopp, G.; Rupp, R.; Moreno, C.; San Cristobal, M.; Boitard, S. Selection signatures in worldwide sheep populations. PLoS ONE 2014, 9, e103813. [CrossRef]

15. Moioli, B.; Pilla, F.; Ciani, E. Signatures of selection identify loci associated with fat tail in sheep. J. Anim. Sci. 2015, 93, 4660-4669. [CrossRef]

16. Pan, Z.; Li, S.; Liu, Q.; Wang, Z.; Zhou, Z.; Di, R.; An, X.; Miao, B.; Wang, X.; Hu, W.; et al. Rapid evolution of a retro-transposable hotspot of ovine genome underlies the alteration of BMP2 expression and development of fat tails. BMC Genom. 2019, 20, 261. [CrossRef]

17. Li, C.; Li, M.; Li, X.; Ni, W.; Xu, Y.; Yao, R.; Wei, B.; Zhang, M.; Li, H.; Zhao, Y.; et al. Whole-Genome Resequencing Reveals Loci Associated With Thoracic Vertebrae Number in Sheep. Front. Genet. 2019, 10. [CrossRef]

18. Li, X.; Ye, J.; Han, X.; Qiao, R.; Li, X.; Lv, G.; Wang, K. Whole-genome sequencing identifies potential candidate genes for reproductive traits in pigs. Genomics 2019. [CrossRef] 
19. Li, W.-T.; Zhang, M.-M.; Li, Q.-G.; Tang, H.; Zhang, L.-F.; Wang, K.-J.; Zhu, M.-Z.; Lu, Y.-F.; Bao, H.-G.; Zhang, Y.-M.; et al. Whole-genome resequencing reveals candidate mutations for pig prolificacy. Proc. R. Soc. B Biol. Sci. 2017, $284,20172437$. [CrossRef]

20. Guan, D.; Luo, N.; Tan, X.; Zhao, Z.; Huang, Y.; Na, R.; Zhang, J.; Zhao, Y. Scanning of selection signature provides a glimpse into important economic traits in goats (Capra hircus). Sci. Rep. 2016, 6, 36372. [CrossRef]

21. Lai, F.-N.; Zhai, H.-L.; Cheng, M.; Ma, J.-Y.; Cheng, S.-F.; Ge, W.; Zhang, G.-L.; Wang, J.-J.; Zhang, R.-Q.; Wang, X.; et al. Whole-genome scanning for the litter size trait associated genes and SNPs under selection in dairy goat (Capra hircus). Sci. Rep. 2016, 6, 38096. [CrossRef] [PubMed]

22. Dolebo, A.T.; Khayatzadeh, N.; Melesse, A.; Wragg, D.; Rekik, M.; Haile, A.; Rischkowsky, B.; Rothschild, M.F.; Mwacharo, J.M. Genome-wide scans identify known and novel regions associated with prolificacy and reproduction traits in a sub-Saharan African indigenous sheep (Ovis aries). Mamm. Genome 2019, 30, 339-352. [CrossRef] [PubMed]

23. Ruiz-Larrañaga, O.; Langa, J.; Rendo, F.; Manzano, C.; Iriondo, M.; Estonba, A. Genomic selection signatures in sheep from the Western Pyrenees. Genet. Sel. Evol. 2018, 50, 9. [CrossRef] [PubMed]

24. Patel, R.K.; Jain, M. NGS QC Toolkit: A Toolkit for Quality Control of Next Generation Sequencing Data. PLoS ONE 2012, 7, e30619. [CrossRef] [PubMed]

25. Li, H.; Durbin, R. Fast and accurate short read alignment with Burrows-Wheeler transform. Bioinformatics 2009, 25, 1754-1760. [CrossRef] [PubMed]

26. Li, H.; Handsaker, B.; Wysoker, A.; Fennell, T.; Ruan, J.; Homer, N.; Marth, G.; Abecasis, G.; Durbin, R.; Proc, G.P.D. The Sequence Alignment/Map format and SAMtools. Bioinformatics 2009, 25, 2078-2079. [CrossRef] [PubMed]

27. Wang, K.; Li, M.Y.; Hakonarson, H. ANNOVAR: Functional annotation of genetic variants from high-throughput sequencing data. Nucleic Acids Res. 2010, 38, e164. [CrossRef]

28. Danecek, P.; Auton, A.; Abecasis, G.; Albers, C.A.; Banks, E.; DePristo, M.A.; Handsaker, R.E.; Lunter, G.; Marth, G.T.; Sherry, S.T.; et al. The variant call format and VCFtools. Bioinformatics 2011, 27, 2156-2158. [CrossRef]

29. Lee, T.H.; Guo, H.; Wang, X.Y.; Kim, C.; Paterson, A.H. SNPhylo: A pipeline to construct a phylogenetic tree from huge SNP data. BMC Genom. 2014, 15, 162. [CrossRef]

30. Barrett, J.C.; Fry, B.; Maller, J.; Daly, M.J. Haploview: Analysis and visualization of LD and haplotype maps. Bioinformatics 2005, 21, 263-265. [CrossRef]

31. Rubin, C.-J.; Zody, M.C.; Eriksson, J.; Meadows, J.R.S.; Sherwood, E.; Webster, M.T.; Jiang, L.; Ingman, M.; Sharpe, T.; Ka, S.; et al. Whole-genome resequencing reveals loci under selection during chicken domestication. Nature 2010, 464, 587-591. [CrossRef] [PubMed]

32. Weir, B.S.; Cockerham, C.C. Estimating F-Statistics for the analysis of population structure. Evolution 1984, 38, 1358-1370. [CrossRef] [PubMed]

33. Stajich, J.E.; Block, D.; Boulez, K.; Brenner, S.E.; Chervitz, S.A.; Dagdigian, C.; Fuellen, G.; Gilbert, J.G.R.; Korf, I.; Lapp, H.; et al. The bioperl toolkit: Perl modules for the life sciences. Genome Res. 2002, 12, 1611-1618. [CrossRef] [PubMed]

34. Axelsson, E.; Ratnakumar, A.; Arendt, M.-L.; Maqbool, K.; Webster, M.T.; Perloski, M.; Liberg, O.; Arnemo, J.M.; Hedhammar, Å.; Lindblad-Toh, K. The genomic signature of dog domestication reveals adaptation to a starch-rich diet. Nature 2013, 495, 360-364. [CrossRef] [PubMed]

35. Scheet, P.; Stephens, M. A fast and flexible statistical model for large-scale population genotype data: Applications to inferring missing genotypes and haplotypic phase. Am. J. Hum. Genet. 2006, 78, 629-644. [CrossRef] [PubMed]

36. Pickrell, J.K.; Coop, G.; Novembre, J.; Kudaravalli, S.; Li, J.Z.; Absher, D.; Srinivasan, B.S.; Barsh, G.S.; Myers, R.M.; Feldman, M.W.; et al. Signals of recent positive selection in a worldwide sample of human populations. Genome Res. 2009, $19,826-837$. [CrossRef]

37. Zhao, J.H. Gap: Genetic Analysis Package. J. Stat. Softw. 2020, 23, 1-18. [CrossRef]

38. Huang, D.W.; Sherman, B.T.; Lempicki, R.A. Systematic and integrative analysis of large gene lists using DAVID bioinformatics resources. Nat. Protoc. 2008, 4, 44. [CrossRef]

39. Song, S.; Tian, D.; Li, C.; Tang, B.; Dong, L.; Xiao, J.; Bao, Y.; Zhao, W.; He, H.; Zhang, Z. Genome Variation Map: A data repository of genome variations in BIG Data Center. Nucleic Acids Res. 2017, 46, D944-D949. [CrossRef]

40. Partners, N.G.D.C.M.A. Database Resources of the National Genomics Data Center in 2020. Nucleic Acids Res. 2019, 48, D24-D33. [CrossRef]

41. Nielsen, R. Molecular Signatures of Natural Selection. Annu. Rev. Genet. 2005, 39, 197-218. [CrossRef]

42. Wu, J.; Jiang, R. Prediction of Deleterious Nonsynonymous Single-Nucleotide Polymorphism for Human Diseases. Sci. World J. 2013, 2013, 10. [CrossRef] [PubMed]

43. Upadhyay, A.K.; Judge, R.A.; Li, L.; Pithawalla, R.; Simanis, J.; Bodelle, P.M.; Marin, V.L.; Henry, R.F.; Petros, A.M.; Sun, C. Targeting lysine specific demethylase 4A (KDM4A) tandem TUDOR domain-A fragment based approach. Bioorganic Med. Chem. Lett. 2018, 28, 1708-1713. [CrossRef] [PubMed]

44. Burke Molly, K. How does adaptation sweep through the genome? Insights from long-term selection experiments. Proc. R. Soc. B Biol. Sci. 2012, 279, 5029-5038. [CrossRef]

45. Johansson, A.M.; Pettersson, M.E.; Siegel, P.B.; Carlborg, Ö. Genome-Wide Effects of Long-Term Divergent Selection. PLoS Genet. 2010, 6, e1001188. [CrossRef] [PubMed] 
46. Sabeti, P.C.; Varilly, P.; Fry, B.; Lohmueller, J.; Hostetter, E.; Cotsapas, C.; Xie, X.; Byrne, E.H.; McCarroll, S.A.; Gaudet, R.; et al. Genome-wide detection and characterization of positive selection in human populations. Nature 2007, 449, 913-918. [CrossRef] [PubMed]

47. Nosrati, M.; Asadollahpour Nanaei, H.; Amiri Ghanatsaman, Z.; Esmailizadeh, A. Whole genome sequence analysis to detect signatures of positive selection for high fecundity in sheep. Reprod. Domest. Anim. Zuchthyg. 2019, 54, 358-364. [CrossRef]

48. Liu, Z.; Ji, Z.; Wang, G.; Chao, T.; Hou, L.; Wang, J. Genome-wide analysis reveals signatures of selection for important traits in domestic sheep from different ecoregions. BMC Genom. 2016, 17, 863. [CrossRef]

49. Clarke, I.J.; Arbabi, L. New concepts of the central control of reproduction, integrating influence of stress, metabolic state, and season. Domest. Anim. Endocrinol. 2016, 56, S165-S179. [CrossRef]

50. Filis, P.; Lannagan, T.; Thomson, A.; Murray, A.A.; Kind, P.C.; Spears, N. Phospholipase C- $\beta 1$ Signaling Affects Reproductive Behavior, Ovulation, and Implantation. Endocrinology 2009, 150, 3259-3266. [CrossRef]

51. Cao, Z.; Fan, R.; Meng, B.; Xing, Z.; Liu, M.; Gao, M.; Luan, X. Comparative proteomic analysis of hypothalamus tissue from Huoyan geese between pre-laying period and laying period using an iTRAQ-based approach. Anim. Sci. J. 2018, 89, 946-955. [CrossRef] [PubMed]

52. Stamatiades, G.A.; Carroll, R.S.; Kaiser, U.B. GnRH-A Key Regulator of FSH. Endocrinology 2018, 160, 57-67. [CrossRef] [PubMed]

53. Jonak, C.R.; Lainez, N.M.; Boehm, U.; Coss, D. GnRH Receptor Expression and Reproductive Function Depend on JUN in GnRH Receptor-Expressing Cells. Endocrinology 2018, 159, 1496-1510. [CrossRef] [PubMed]

54. Wilson, C.; Krieg, A.J. KDM4B: A Nail for Every Hammer? Genes 2019, 10, 134. [CrossRef]

55. Gaughan, L.; Stockley, J.; Coffey, K.; O’Neill, D.; Jones, D.L.; Wade, M.; Wright, J.; Moore, M.; Tse, S.; Rogerson, L.; et al. KDM4B is a Master Regulator of the Estrogen Receptor Signalling Cascade. Nucleic Acids Res. 2013, 41, 6892-6904. [CrossRef]

56. Krieg, A.J.; Mullinax, S.R.; Grimstad, F.; Marquis, K.; Constance, E.; Hong, Y.; Krieg, S.A.; Roby, K.F. Histone demethylase KDM4A and KDM4B expression in granulosa cells from women undergoing in vitro fertilization. J. Assist. Reprod. Genet. 2018, 35, 993-1003. [CrossRef]

57. Krieg, S.A.; Fan, X.; Hong, Y.; Sang, Q.-X.; Giaccia, A.; Westphal, L.M.; Lathi, R.B.; Krieg, A.J.; Nayak, N.R. Global alteration in gene expression profiles of deciduas from women with idiopathic recurrent pregnancy loss. Mol. Hum. Reprod. 2012, 18, 442-450. [CrossRef] 